\title{
Molecular pathogenesis of leukemia and leukemic stem cells (LSCs)
}

\author{
Masahiro Kizaki ${ }^{1}$
}

Received: 1 October 2018 / Accepted: 9 October 2018 / Published online: 23 October 2018

(c) The Japanese Society of Hematology 2018

The 79th Annual Meeting of the Japanese Society of Hematology (JSH) was held over a 3-day period from Friday, October 20 to Sunday, October 22, 2017. This meeting was the first time in 18 years that the congress was held in Tokyo. Recent years have seen dramatic advances in hematology. Advances in basic research, mainly molecular biology and stem cell research, have enabled the identification of the molecular pathogenesis of leukemia, and the introduction of new therapeutic methods based on these discoveries has greatly transformed the clinical management of leukemia. As a president of the annual meeting, I selected "Innovation and Creation: Advances in Hematology for the Next Generation" as the theme of the congress. "Innovation and Creation" are important topics that are now required of us in studying the development of hematology. In addition, internationalization and the focus on the young generation have been the most important missions of the JSH. Therefore, along with this theme, I organized a Presidential Symposium with the title "Young Japanese Hematologists in the World: Molecular Pathogenesis of Leukemia and Leukemic Stem Cells (LSCs)"; in this symposium, five young Japanese researchers who are active on the global stage, individuals who serve as principal investigators (PIs) with their own laboratories discussed the molecular pathogenesis of leukemia and leukemic stem cells. I am sure that these enthusiastic presentations by successful young researchers from the global front lines echoed in the hearts of the younger generation in Japan. To commemorate this exciting symposium, I invited four speakers from congress to review their topics in the session of the "Progress in Hematology" in this issue of the International Journal of Hematology (IJH).

Masahiro Kizaki

makizaki@saitama-med.ac.jp

1 Department of Hematology, Saitama Medical Center, Saitama Medical University, 1981 Kamoda, Kawagoe, Saitama 350-8550, Japan
Transcription factors play a central role in the regulation of hematopoiesis. Molecular lesions in genes encoding transcription factors are common oncogenic events in leukemia. Perturbations in transcription factor activity result in a differentiation block, cellular proliferation, lineage infidelity, and transformation events that lead to the activation of various signaling pathways in leukemic cells [1]. The complexity of the transcription process offers a large number of substrates for designing therapeutic targets; however, therapies targeting transcription factors in leukemia can be effective as exemplified by the use of tyrosine kinase inhibitors for the treatment of chronic myeloid leukemia (CML) [2].

Dr. Takaomi Sanda and his colleague from the Cancer Institute of Singapore, National University of Singapore, discussed the transcriptional regulatory network and downstream target genes, including protein-coding genes and noncoding RNAs, that are controlled by TAL1 during normal hematopoiesis and T-cell leukemogenesis [3]. TAL1 has important oncogenic properties in T-acute lymphoblastic leukemia (T-ALL), and the authors investigated the role of the oncogenic transcription factor TAL1 and its regulatory partners such as GATA3, RUNX1, and MYB, which are encoded by genes that are regulated by super-enhancers [4]. Furthermore, those authors continue to seek the true molecular targets of TAL1 and to develop the novel therapeutic approaches for the treatment of T-ALL.

Dr. Susumu Kobayashi and his colleague from the Beth Israel Deaconess Center and Harvard Medical School have been investigating the genetic and epigenetic mechanisms of regulation of transcription factors in acute myeloid leukemia (AML). In this review article, Kobayashi et al. discussed on the dysfunction of transcription factors such as those encoded by $C / E B P \alpha, P U .1$, and $R U N X 1$, effects mediated by gene mutations, chromosomal aberrations, or the elimination of expression, thereby leading to the development of AML [5]. In addition, they report their identification by highthroughput screening of the novel small molecule ICCB280, a quinazolinone derivative, as an inducer of $C / E B P \alpha$ [6]. 
ICCB280 induces differentiation of myeloid leukemic cells, leading the Kobayashi laboratory to propose that ICCB280 might serve as a novel AML therapy targeting a myeloidspecific transcription factor.

Recent research progress has clarified that the biology of LSCs, providing insight on the self-renewal capacity, stemness properties, and drug resistance of LSCs, properties that contribute to the leukemia development and relapse [7]. Therefore, understanding the molecular machineries essential for LSC and identification of the specific target molecules for LSCs will be important issues for the basic and clinical fields of hematology.

Dr. Keisuke Ito and his colleagues from the Albert Einstein College of Medicine summarized their recent progress on the metabolic regulation of cell fate decision in hematopoietic stem cells (HSCs); they also discussed their work on key metabolic pathways of maintaining stemness of LSCs, and on therapeutic approaches targeting leukemia metabolism [8]. To understand the mechanisms of HSC fate decision, Ito at al. have developed and performed analyses on highly purified HSCs and single cells. Their work demonstrated that mitochondrial metabolism is a key regulator of cell fate choice, indicating that the quality of mitochondria is important to maintaining the equilibrium of HSC populations [9]. This new concept is expected to lead to novel therapeutic approaches that use metabolic manipulations for the treatment of leukemia and other hematological malignancies.

Finally, Dr. Momoko Yoshimoto and her colleagues from the University of Texas Health Science Center at Houston focused on the origin of HSCs in the mouse embryo and introduced a view of native hematopoiesis and possible leukemia-initiating cells, including their original investigations $[10,11]$. The origin of HSCs has been an interest of research in the filed of developmental biology, and the authors reviewed their use of lineage tracing to address this issue. These authors used the same method to evaluate the development of prenatal leukemia-initiating cells, concluding that the combination of lineage tracing assays and in vivo clonal assays will improve our understanding of the kinetics of HSCs and leukemia-initiating cells that emerge during ontogeny.

This symposium was chaired by Dr. Scott S. Armstrong from the Dana-Farber Cancer Institute and Dr. Stephen D. Nimer from the University of Miami. The four speakers presented their recent research progress and the symposium itself proceeded at a high scientific level with active discussion. As the president of the JSH2017, I was proud of the four distinguished young Japanese hematologists who are employed around the world; I am sure that this symposium had a large influence on young hematologists working throughout Japan. I spared no effort to make the 79th Annual Meeting of the JSH an academically fulfilling conference that would be useful for future clinical practice and research, as well as an enjoyable event.

Finally, I hope that this special issue of the IJH provides a practical introduction to the attractive and developing field of the molecular pathogenesis of leukemia and LSCs. In addition, I thank the four outstanding young hematologists who spoke at this symposium for their contributions to the JSH, and look forward to their ongoing scientific development and success.

\section{References}

1. Rosenbauer F, Tenen DG. Transcription factors in myeloid development: balancing differentiation with transformation. Nat Rev Immunol. 2007;7:105-17.

2. Steegmann JL, Baccarani M, Breccia M, Casado LF, GarcíaGutiérrez V, Hochhaus A, et al. European LeukemiaNet recommendations for the management and avoidance of adverse events of treatment in chronic myeloid leukaemia. Leukemia. 2016;30:1648-71.

3. Ngoc PCT, Tan SH, Tan TK, Chan MM, Li Z, Yeoh AEJ. at al. Identification of novel IncRNA regulated by the TAL1 complex in T-cell acute lymphoblastic leukemia. Leukemia. 2018. https:// doi.org/10.1038/s41375-018-0110-4 (Epub ahead of print).

4. Sanda T, Leong WZ. TAL1 as a master oncogeneic transcription factor in T-cell acute lymphoblastic leukemia. Exp Hematol. 2017;53:7-15.

5. Look AT. Oncogenic transcription factors in the human acute leukemias. Science. 1997;278:1059-64.

6. Radmaska HS, Jernigan F, Nakayama S, Jorge SE, Sun L, Tenen DG. at al. J Biomol Screen. 2015;20:1150-9.

7. O'Brien JA, Rizzieri DA. Leukemic stem cells: a review. Cancer Invest. 2013;31:215-20.

8. Ito K, Suda T. Metabolic requirements for the maintenance of self-renewing stem cells. Nat Rev Mol Cell Biol. 2014;15:243-56.

9. Ito K, Ito K. Hematopoietic stem cell fate through metabolic control. Exp Hematol. 2018;64:1-11.

10. Yoshimoto M, Montecino-Rodriguez E, Ferkowicz MJ, Porayette P, Shelley WC, Conway SJ, et al. Embryonic day 9 yolk sac and intra-embryonic hemogenic endothelium independently generate a B-1 and marginal zone progenitor lacking B-2 potential. Proc Natl Acad Sci USA. 2011;108:1468-73.

11. Yoshimoto M, Porayette P, Glosson NL, Conway SJ, Carlesso N, Cardoso AA, et al. Autonomous murine T-cell progenitor production in the extra-embryonic yolk sac before HSC emergence. Blood. 2012;119:5706-14. 